\title{
Le carcinome urothélial plasmocytoïde de la vessie : une entité histologique rare au pronostic sombre
}

\author{
Mohamed Hicham Benazzouz, MD ; Younes Essatara, MD ; Hachem Elsayegh, MD ; Ali Iken, MD ; \\ Lounis Benslimane, MD ; Kaoutar Znati, MD ; Yassine Nouini, MD
}

Service d'Urologie « A » Centre hospitalier universitaire lbn Sina Rabat, Morocco

Cite as: Can Urol Assoc J 2014;8(9-10):e733-8. http://dx.doi.org/10.5489/cuaj.2017

Published online October 22, 2014.

\section{Résumé}

Introduction : Le carcinome urothélial plasmocytoïde est une variante histologique rare du carcinome urothélial. Seule une centaine de cas ont été décrits dans la littérature. Dans cette étude, nous faisons état de deux nouvelles observations. En combinant nos résultats à ceux des différentes séries décrites dans la littérature, nous tentons de définir les caractéristiques cliniques et pathologiques ainsi que l'approche thérapeutique de cette pathologie.

Observations : Deux nouveaux cas de carcinome urothélial plasmocytoïde ont été diagnostiqués et pris en charge dans notre établissement. Les 2 patients étaient des hommes de 76 ans en moyenne. L'hématurie était le principal symptôme. Les deux patients ont subi une résection transurétrale de la vessie. Le stade tumoral au moment du diagnostic était avancé dans les deux cas (respectivement T3N0M0 et T3N1M0). Les deux patients ont été traités par cystoprostatectomie. L'analyse histologique de la pièce opératoire, complétée par une étude immunohistochimique, a confirmé le diagnostic de carcinome urothélial plasmocytoïde. Le premier patient est décédé un mois plus tard des suites d'une embolie pulmonaire. Le deuxième patient est décédé au bout de deux mois après avoir reçu deux cycles de chimiothérapie adjuvante. Les données recueillies sur les différentes séries décrites dans la littérature vont dans le même sens que nos données, à savoir un stade avancé au moment du diagnostic et un pronostic sombre.

Conclusion : Le carcinome urothélial plasmocytoïde est une variante histologique rare et agressive du carcinome urothélial. Le diagnostic se fait souvent à un stade avancé, et le pronostic est peu encourageant. Le traitement repose le plus souvent sur une cystectomie, suivie d'une chimiothérapie adjuvante à base de cisplatine. L'intérêt d'une chimiothérapie néo-adjuvante n'a pas encore été établi.

\section{Introduction}

Le carcinome urothélial plasmocytoïde de la vessie est une variante histologique rare du carcinome urothélial qui figure depuis 2004 dans la classification des tumeurs établie par I'OMS'. Dans la littérature, seule une centaine de cas ont été décrits à ce jour. Les données disponibles suggèrent que ce type histologique est agressif et le plus souvent diagnostiqué à un stade avancé. Nous faisons état de deux nouveaux cas de carcinome urothélial plasmocytö̈de de la vessie, en plus de procéder à une revue de la littérature et à une analyse des différentes séries traitant de ce sujet.

\section{Observations}

\section{Cas 1}

Un homme âgé de 79 ans, agriculteur de profession sans antécédents pathologiques particuliers, présentait depuis 6 mois une hématurie totale caillotante intermittente, associée à des douleurs hypogastriques et évoluant dans un contexte d'altération de l'état général. L'examen clinique n'a révélé aucune particularité. L'échographie rénale et vésicale (fig. 1) a démontré la présence de 2 lésions hyperéchogènes bourgeonnantes dans la lumière vésicale mesurant respectivement $13 \times 15 \mathrm{~mm}$ (paroi latérale droite) et $30 \times 42 \mathrm{~mm}$ (paroi antérolatérale gauche), sans incidence sur le haut appareil urinaire.

La cystoscopie a révélé la présence d'une énorme tumeur d'allure solide couvrant toute la face latérale gauche et s'étendant au dôme vésical. Devant l'impossibilité de procéder à une chirurgie par endoscopie, seule une résection biopsique profonde a été réalisée. L'analyse anatomopathologique (figs. 2, 3 et 4) a indiqué une prolifération carcinomateuse faite de cellules arrondies non jointives d'aspect plasmocytoïde au cytoplasme éosinophile et au noyau excentré. Cette prolifération infiltrait largement le muscle avec présence d'emboles vasculaires tumoraux. Un complément d'étude immunohistochimique a été réalisé (tableau 1). Le diagnostic de carcinome urothélial plasmocytoïde infiltrant (pT2) de haut grade a été retenu.

Devant le caractère infiltrant de la tumeur, un bilan d'extension par TDM thoraco-abdomino-pelvienne a été réalisé. 


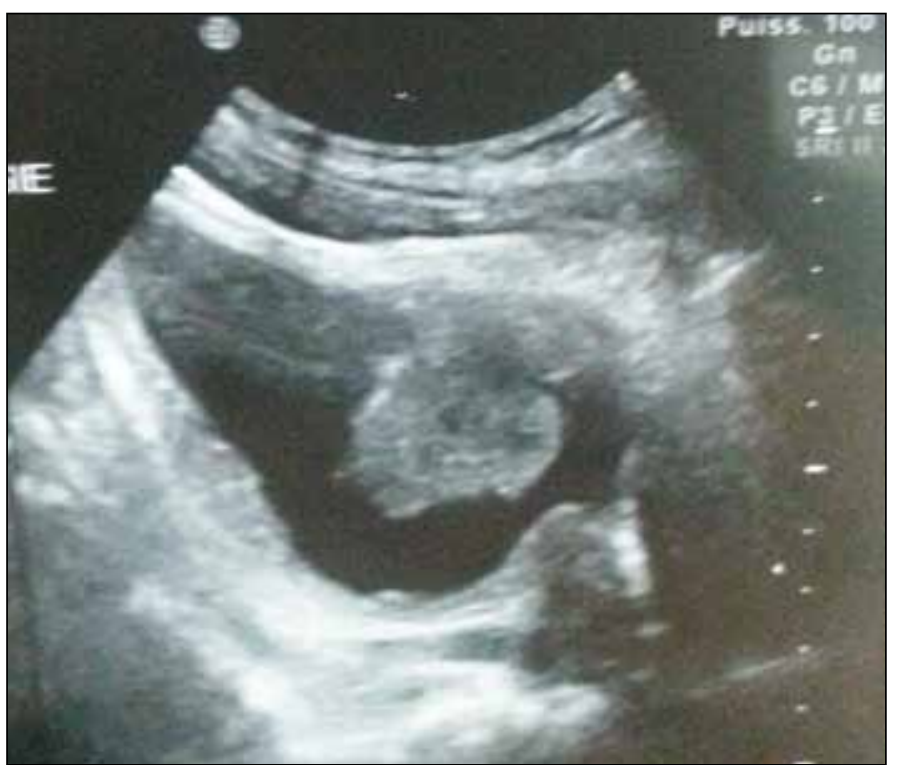

Fig. 1. Échographie vésicale : lésion hyperéchogène de la paroi antérolatérale gauche.

Celui-ci a révélé une vessie siège d'un épaississement pariétal irrégulier mesurant jusqu'à $35 \mathrm{~mm}$, intéressant la paroi latérale gauche et débordant sur le plancher et le dôme (fig. 5). Ce processus infiltrait la graisse périvésicale gauche. Aucune adénopathie ni métastase à distance n'a été décelée, et la tumeur a été classée T3N0M0.

Le patient a subi une cystoprostatectomie totale avec curage ganglionnaire ilio-obturateur bilatéral et une dérivation urinaire de type Bricker. Il s'est écoulé 56 jours entre le diagnostic et la chirurgie. L'analyse anatomopathologique de la pièce opératoire a révélé à la macroscopie une tumeur

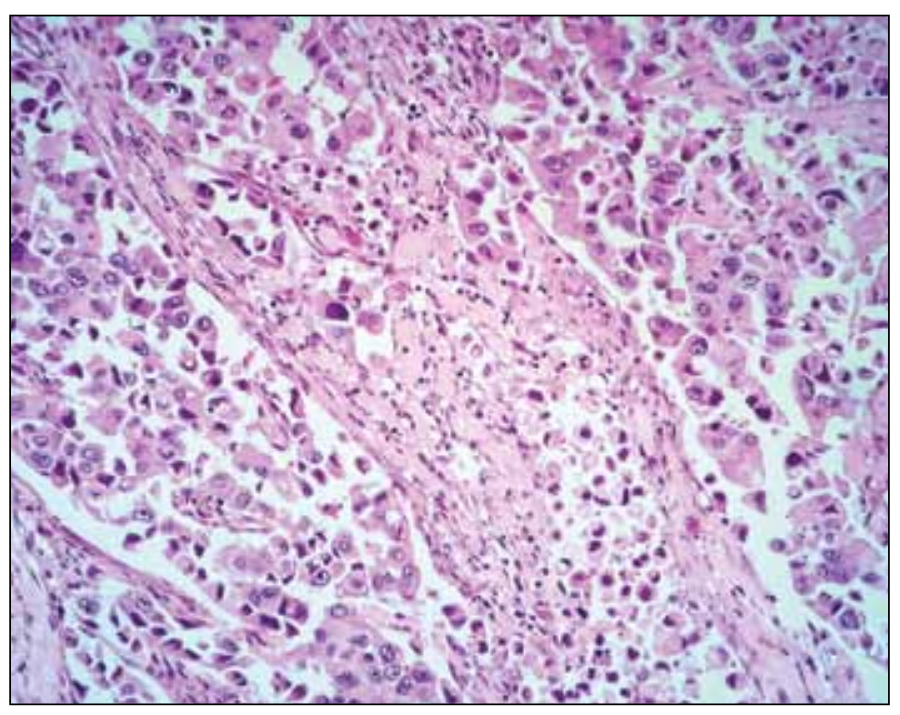

Fig. 2. Prolifération tumorale faite d'un contingent à cellules plasmocytoïdes formant des cordons de cellules disjointives (HE $\times 100$ ).

bourgeonnante et infiltrante de consistance ferme et blanchâtre (fig. 6).

À la microscopie, on a décelé une tumeur infiltrant la paroi vésicale jusqu'à la sous-séreuse et confirmé le diagnostic de carcinome urothélial plasmocytö̈de. Le curage ganglionnaire n'a pas révélé d'envahissement des ganglions (11 ganglions). Les marges chirurgicales étaient négatives. La tumeur a été classée pT3aN0M0-R0. Les suites opératoires ont été simples, et le patient a quitté I'hôpital huit jours après I'intervention. Il est décédé un mois plus tard des suites d'une embolie pulmonaire.

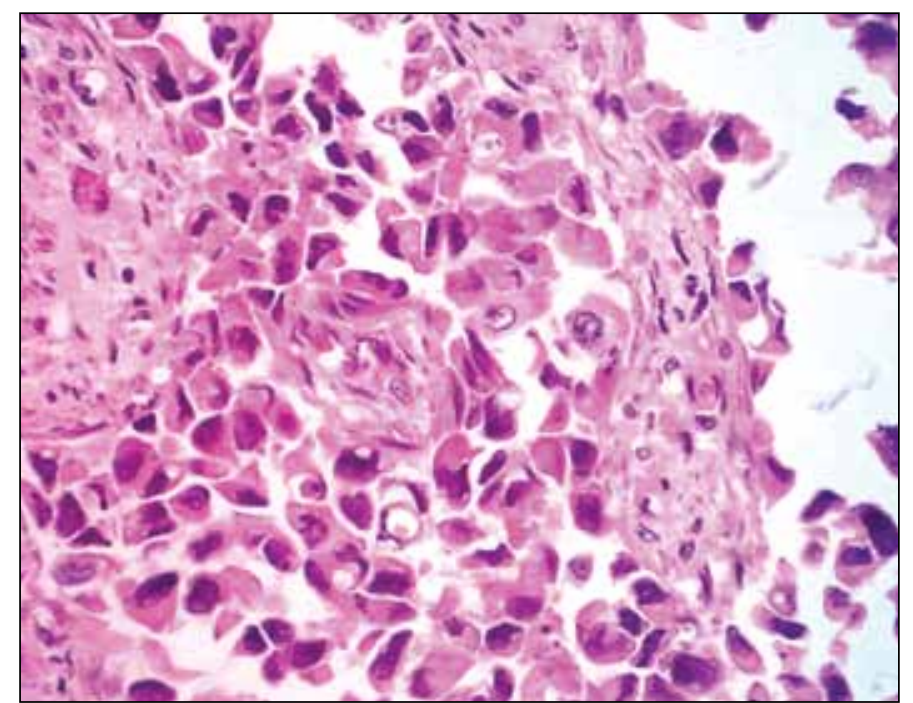

Fig. 3. Cellules plasmocytoïdes comportant des noyaux excentrés fortement atypiques et un cytoplasme éosinophile (HE $\times 400)$.

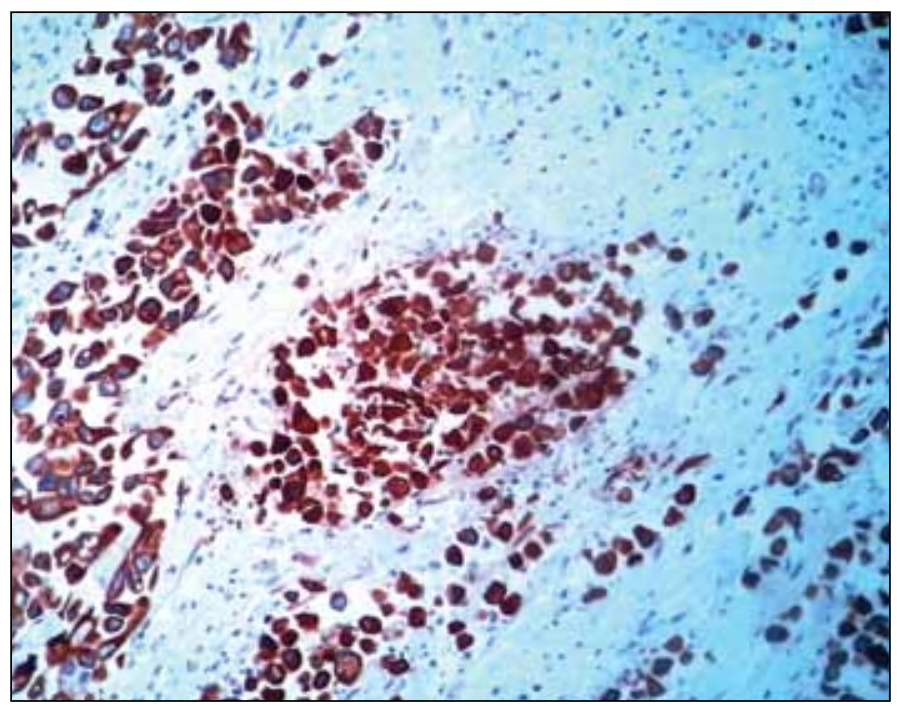

Fig. 4. Expression franche de la cytokératine AE1/AE3 par les cellules tumorales. 


\begin{tabular}{lcc}
\hline \multicolumn{2}{l}{ Tableau 1. Profil immunohistochimique de nos deux patients } \\
\hline Anticorps & Cas 1 & Cas 2 \\
\hline AE1/AE3 & + & + \\
CK7 & + & + \\
CK20 & + & - \\
CD138 & + (expression focale) & + (expression focale) \\
CD79a & - & - \\
LCA & - & - \\
EMA & + & + \\
$\boldsymbol{\lambda}-\mathbf{k}$ & - & - \\
\hline
\end{tabular}

\section{Cas 2}

Ce patient de 73 ans, fumeur chronique, présentait depuis 1 an une hématurie totale caillotante intermittente évoluant dans un contexte d'altération de l'état général. L'examen clinique n'a révélé aucune particularité. Une résection biopsique de la vessie, réalisée dans un autre établissement, a indiqué à l'examen anatomopathologique la présence d'un carcinome urothélial infiltrant pT2 de haut grade avec extension jusqu'à l'urètre prostatique sous forme de carcinome in situ. La TDM thoraco-abdomino-pelvienne réalisée dans le cadre du bilan d'extension a mis en lumière un processus intéressant la paroi vésicale latérale gauche avec envahissement du méat urétéral gauche et urétéro-hydronéphrose d'amont. Ce processus infiltrait la graisse périvésicale avec présence d'adénopathies hypogastriques gauches de $10 \mathrm{~mm}$. La TDM n'a pas indiqué de métastases à distance, et la tumeur a été classée T3N1M0.

Une intervention radicale a été programmée. Il s'est écoulé 70 jours entre le diagnostic et la chirurgie. À l'exploration, on a trouvé des métastases hépatiques. Une cystoprostatectomie totale avec urétrectomie et dérivation urinaire de type urétérostomie cutanée a tout de même été réalisée

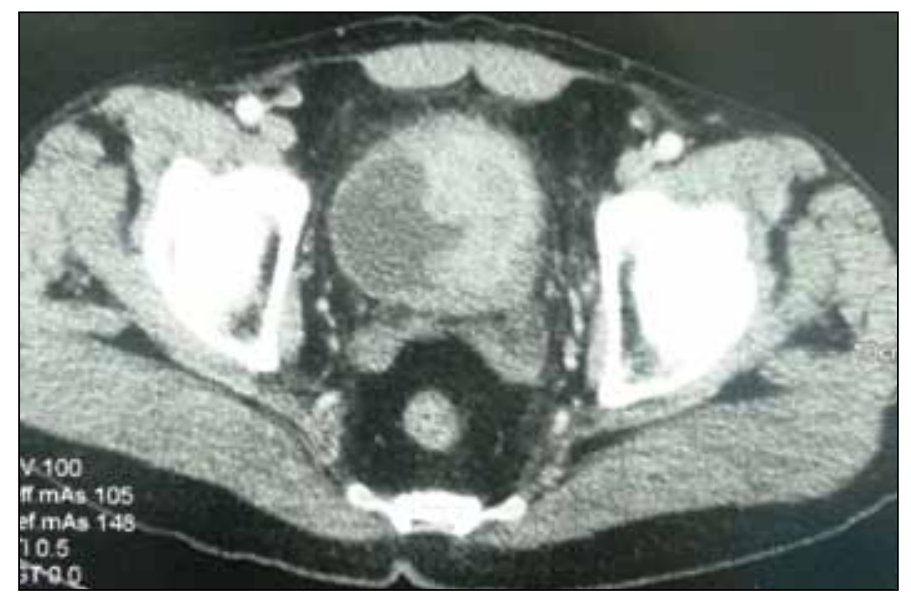

Fig. 5. Coupe tomodensitométrique montrant un épaississement de la paroi vésicale latérale gauche infiltrant la graisse périvésicale. dans un but hémostatique. Les résultats de l'étude anatomopathologique avec complément immunohistochimique (tableau 1) penchaient en faveur d'un carcinome urothélial plasmocytoïde infiltrant toute la paroi vésicale jusqu'à la graisse périvésicale avec extension à la prostate. Le curage ganglionnaire a révélé l'envahissement de trois ganglions sur six du côté gauche, mais d'aucun du côté droit. Les marges chirurgicales et les recoupes urétérales droite et gauche étaient saines. La tumeur a été classée pT4aN2M1-R0.

Les suites opératoires ont été simples, et le patient a obtenu son congé de l'hôpital 10 jours après l'intervention. II a reçu par la suite une chimiothérapie de type M-VAC (méthotrexate, vinblastine, doxorubicine et cisplatine). Cette chimiothérapie n'a pu être poursuivie au-delà du deuxième cycle en raison de l'altération profonde de l'état général du patient qui l'a emporté trois mois après la chirurgie.

\section{Discussion}

Le carcinome urothélial plasmocytoïde de la vessie est une variante histologique rare du carcinome urothélial. Ce type histologique a été décrit la première fois par Sahin et coll. En $1991^{2}$ et depuis lors, seule une centaine de cas ont été signalés dans la littérature, qu'il s'agisse de cas isolés ou de séries de patients, dont la plus vaste comptait 32 patients $^{3}$. Les caractéristiques cliniques et pathologiques de ces patients sont présentées dans le tableau 2.

Selon les données de ce tableau, l'âge moyen au moment du diagnostic est de 64,77 ans, avec une nette prédominance masculine (100 hommes pour 14 femmes). La symptomatologie clinique n'est pas spécifique, mais elle est dominée par une hématurie, associée ou non à des signes irritatifs du bas appareil urinaire. À la cystoscopie, la tumeur peut

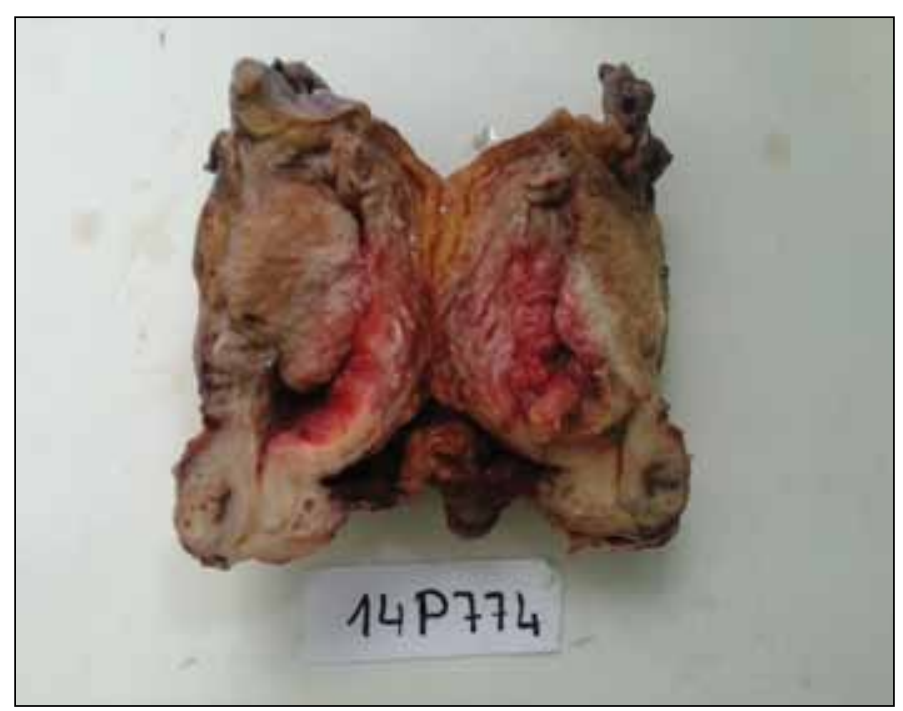

Fig. 6. Macroscopie de la pièce cystectomique : à la coupe, présence d'une tumeur vésicale bourgeonnante et infiltrante de couleur blanchâtre. 


\begin{tabular}{|c|c|c|c|c|c|c|}
\hline Série & Nb & Âge / sexe & Symptômes & TNM & Traitement & Évolution et suivi \\
\hline Sahin $^{1}$ & 2 & $\begin{array}{l}-68 \text { ans } \\
-\mathrm{H}: 2 \\
-\mathrm{F}: 0\end{array}$ & $-N D$ & $\begin{array}{l}-\mathrm{T} 2: 1 \\
-\mathrm{ND}: 1\end{array}$ & $\begin{array}{l}\text { - Chimiothérapie : } 1 \\
\text { - Traitement palliatif : } 1\end{array}$ & $\begin{array}{c}\text { - VSM : } 1 \text { (24 mois) } \\
\text { - PDV : } 1\end{array}$ \\
\hline Zukerberg $^{2}$ & 1 & $\begin{array}{l}-68 \text { ans } \\
-H: 1\end{array}$ & - ND & T2 & -Radiochimiothérapie & - VSM (17 mois) \\
\hline $\mathrm{Ro}^{3}$ & 9 & $\begin{array}{l}-64,3 \text { ans } \\
-H: 9 \\
-F: 0\end{array}$ & $\begin{array}{l}\text { - Hématurie : } 8 \\
\text { - Urgenturie : } 1\end{array}$ & $\begin{array}{l}-\mathrm{T} 1: 1 \\
-\mathrm{T} 2: 2 \\
-\mathrm{T} 3: 5 \\
-\mathrm{T} 4: 1\end{array}$ & $\begin{array}{c}\text { - Cystectomie + chimiothérapie } \\
: 4 \\
\text { - Cystectomie : } 2 \\
\text { - Chimiothérapie : } 1 \\
\text { - Instillation de BCG : } 1 \\
\text { - Aucun traitement : } 1\end{array}$ & $\begin{array}{l}\text { - DCM : } 5 \text { (5-36 mois) } \\
\text { - VAM : } 3 \text { (30-47 mois) } \\
\text { - PDV : } 1\end{array}$ \\
\hline Gaafar ${ }^{4}$ & 7 & $\begin{array}{l}-69,1 \text { ans } \\
-H: 7 \\
-F: 0\end{array}$ & - Hématurie : 7 & $-\mathrm{T} 3: 7$ & $\begin{array}{c}\text { - RTUV + chimiothérapie : } 5 \\
\text { - Cystectomie + chimiothérapie } \\
: 2\end{array}$ & $\begin{array}{l}\text { - DCM : } 3 \text { (à } 24 \text { mois) } \\
\text { - VAM :4 (4-24 mois) }\end{array}$ \\
\hline Lopez-Beltran ${ }^{5}$ & 11 & $\begin{array}{l}-67 \text { ans } \\
H: 9 \\
-F: 2\end{array}$ & - Hématurie : 11 & $\begin{array}{l}-\mathrm{T} 3 \mathrm{~N} 0: 3 \\
-\mathrm{T} 3 \mathrm{~N} 1: 3 \\
-\mathrm{T} 3 \mathrm{~N} 2: 1 \\
-\mathrm{T} 4 \mathrm{~N} 1: 4\end{array}$ & $\begin{array}{c}\text { - Cystectomie + chimiothérapie } \\
: 5 \\
\text { - RTUV + chimiothérapie : } 6\end{array}$ & $\begin{array}{l}\text { - DCM : } 9 \text { (2-11 mois) } \\
\text { - VAM : } 2 \text { (8-16 mois) }\end{array}$ \\
\hline Nigwekar 6 & 17 & $\begin{array}{l}-70 \text { ans } \\
-H: 15 \\
-F: 2\end{array}$ & $\begin{array}{l}\text { - Hématurie : } 15 \\
\text { - ND : } 2\end{array}$ & 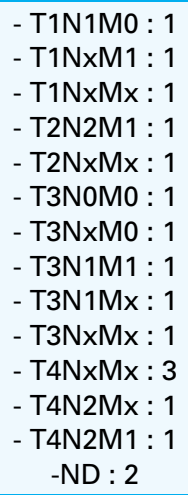 & $\begin{array}{c}\text { - Cystectomie : } 6 \\
\text { - Biopsie }: 3 \\
\text { - Cystectomie + chimiothérapie } \\
: 1 \\
\text { - RTUV + chimiothérapie : } 7\end{array}$ & $\begin{array}{l}\text { - DCM : } 11(0,5-43 \\
\text { mois) } \\
\text { - VAM : } 5 \text { (4-9 mois) } \\
\text { - ND : } 1\end{array}$ \\
\hline Fritsche $^{7}$ & 5 & $\begin{array}{l}-66,2 \text { ans } \\
-H: 4 \\
-F: 1\end{array}$ & $\begin{array}{l}\text { - Hématurie : } 1 \\
\text { - Signes irritatifs } \\
: 4\end{array}$ & $\begin{array}{l}\text { - T3NOM0 : } 1 \\
- \text { T4NOM0 : } 2 \\
- \text { T4N2M0 : } 1 \\
\text { - ND : } 1\end{array}$ & $\begin{array}{c}\text { - Cystectomie : } 1 \\
\text { - Cystectomie + chimiothérapie } \\
: 2 \\
\text { - RTUV + chimiothérapie : } 1 \\
\text { - RTUV + BCGthérapie : } 1\end{array}$ & $\begin{aligned} \text { - DCM } & \text { : } 2 \text { (14-29 mois) } \\
& - \text { ND : } 3\end{aligned}$ \\
\hline $\mathrm{Keck}^{8}$ & 32 & $\begin{array}{l}-\mathrm{ND}: 16 \\
-56,9 \text { ans } \\
-\mathrm{H}: 13 \\
-\mathrm{F}: 3\end{array}$ & $-N D$ & $\begin{array}{l}-\mathrm{T} 2 \mathrm{~N}+: 2 \\
-\mathrm{T} 3 \mathrm{~N} 0: 4 \\
-\mathrm{T} 3 \mathrm{~N}+: 7 \\
-\mathrm{T} 4 \mathrm{~N} 0: 1 \\
-\mathrm{T} 4 \mathrm{~N}+: 2 \\
-\mathrm{ND}: 16\end{array}$ & $\begin{array}{l}\text { - Cystectomie + chimiothérapie } \\
: 16 \\
\text { - ND : } 16\end{array}$ & $\begin{array}{l}\text { Médiane de survie } \\
\text { globale : } 23,4 \text { mois }\end{array}$ \\
\hline Dayyani $^{9}$ & 31 & $\begin{array}{l}-63 \text { ans } \\
-H: 27 \\
-F: 4\end{array}$ & $-N D$ & $\begin{array}{l}\text { - T1N0M0 : } 4 \\
\text { - T2N0M0 : } 7 \\
\text { - T3N0M0 : } 4 \\
\text { - T4N0M0 : } 8 \\
\text { - TxN1M0 : } 4 \\
\text { - TxNxM1:4 }\end{array}$ & $\begin{array}{c}\text { - Chirurgie : } \\
\text { *ND : } 1 \\
\text { *Cystectomie : } 17 \\
{ }^{*} \text { Exentération : } 2 \\
{ }^{*} \text { RTUV : } 2 \\
{ }^{*} \text { Abstention : } 9 \\
\text { - Chimiothérapie : } \\
\text { *Néo-adjuvante : } 7 \\
\text { *Adjuvante }: 7 \\
\text { * Métastatique : } 14 \\
\text { *Abstention : } 3\end{array}$ & $\begin{array}{l}\text { Médiane de survie } \\
\text { globale : } 17,7 \text { mois } \\
\text { (45,8 mois pour } \\
\text { les patients non } \\
\text { métastatiques [stade I- } \\
\text { III] c. } 13,4 \text { mois pour le } \\
\text { stade IV) }\end{array}$ \\
\hline
\end{tabular}




\begin{tabular}{|c|c|c|c|c|c|c|}
\hline Série & $\mathbf{N b}$ & Âge / sexe & Symptômes & TNM & Traitement & Évolution et suivi \\
\hline $\mathrm{Mai}^{10}$ & 7 & $\begin{array}{l}-69 \text { ans } \\
-N D: 6 \\
-F: 1\end{array}$ & $\begin{array}{l}\text { - Douleur } \\
\text { hypogastrique } \\
: 1 \\
\text { - ND : } 6\end{array}$ & $-N D$ & $\begin{array}{l}\text { - Cystectomie : } 1 \\
\text { - ND : } 6\end{array}$ & $\begin{array}{l}\text { - DCM : } 1 \text { (12 mois) } \\
\text { - ND : } 6\end{array}$ \\
\hline Kohno ${ }^{11}$ & 1 & $\begin{array}{l}-76 \text { ans } \\
-\mathrm{H}: 1\end{array}$ & $\begin{array}{l}\text { - Brûlures } \\
\text { mictionnelles }\end{array}$ & $-\mathrm{T} 4$ & - Cystectomie + chimiothérapie & - VSM (36 mois) \\
\hline Mitsogiannis ${ }^{12}$ & 1 & $\begin{array}{l}-60 \text { ans } \\
-\mathrm{H}: 1\end{array}$ & - Hématurie & $-\mathrm{T3}$ & - Biopsie & - DCM (7 jours) \\
\hline Aldousari ${ }^{13}$ & 1 & $\begin{array}{l}-57 \text { ans } \\
-\mathrm{H}: 1\end{array}$ & - Hématurie & $-\mathrm{T3}$ & - Cystectomie + chimiothérapie & - DCM (6 mois) \\
\hline Sato $^{14}$ & 1 & $\begin{array}{l}-50 \text { ans } \\
-\mathrm{H}: 1\end{array}$ & - Pollakiurie & $-\mathrm{T} 4$ & - Cystectomie & - DCM (24 mois) \\
\hline Sakuma ${ }^{15}$ & 2 & $\begin{array}{l}-72,5 \text { ans } \\
-\mathrm{H}: 2 \\
-\mathrm{F}: 0\end{array}$ & $\begin{array}{l}\text { - Hématurie : } 1 \\
\text { - Urgenturie : } 1\end{array}$ & - T3 : 2 & $\begin{array}{c}\text { - Cystectomie }+ \text { chimiothérapie } \\
: 2\end{array}$ & $\begin{array}{l}\text { - DCM : } 1 \text { (24 mois) } \\
\text { - VSM : } 1 \text { (16 mois) }\end{array}$ \\
\hline Shimada ${ }^{16}$ & 1 & $\begin{array}{l}-46 \text { ans } \\
-\mathrm{H}: 1\end{array}$ & - Hématurie & $-N D$ & $\begin{array}{c}\text { - RTUV + radiothérapie + } \\
\text { chimiothérapie }\end{array}$ & - DCM (8 mois) \\
\hline Soylu ${ }^{17}$ & 1 & $\begin{array}{l}-67 \text { ans } \\
-\mathrm{H}: 1\end{array}$ & - Hématurie & - T2 & - Cystectomie de sauvetage & - DCM (18 mois) \\
\hline Raspollini ${ }^{18}$ & 4 & $\begin{array}{l}-68,5 \text { ans } \\
-H: 3 \\
-F: 1\end{array}$ & $\begin{array}{l}\text { - Hématurie : } 3 \\
\text { - Pollakiurie : } 1\end{array}$ & $\begin{array}{l}- \text { T3N0M0 }: 1 \\
- \text { T3N1M0 }: 1 \\
- \text { T3N1M1 }: 1 \\
- \text { T4N0M0 : } 1\end{array}$ & $\begin{array}{c}\text { - Cystectomie }: 3 \\
\text { - Cystectomie }+ \text { chimiothérapie } \\
: 1\end{array}$ & $\begin{array}{l}\text { - DCM : } 3 \text { (6-10 mois) } \\
\text { - VAM : } 1 \text { (18 mois) }\end{array}$ \\
\hline Notre série & 2 & $\begin{array}{l}-76 \text { ans } \\
-\mathrm{H}: 2\end{array}$ & - Hématurie : 2 & $\begin{array}{l}\text { - T3NOM0 } \\
\text { - T3N1M0 }\end{array}$ & $\begin{array}{c}\text { - Cystectomie } \\
\text { - Cystectomie }+ \text { chimiothérapie } \\
: 1\end{array}$ & - DCM : 2 (1 et 3 mois) \\
\hline
\end{tabular}

$\mathrm{H}$ : homme ; $\mathrm{F}$ : femme ; ND : non documenté ; RTUV : résection transurétrale de vessie ; DCM : décès causé par la maladie ; VAM : vivant avec la maladie ; VSM : vivant sans la maladie.

être unique ou multiple et peut siéger n'importe où dans la vessie. Par conséquent, le diagnostic de carcinome urothélial plasmocytoïde est purement histologique, avec présence de petites cellules à noyau excentré hyperchromatique et dont le cytoplasme abondant éosinophile renferme de très petites vacuoles de mucus ressemblant à des plasmocytes ${ }^{4}$. Cette morphologie cellulaire plasmocytoïde peut être également observée dans d'autres tumeurs vésicales comme les lymphomes, les plasmocytomes, les carcinomes neuroendocrines, les rhabdomyosarcomes, les myélomes multiples, les métastases vésicales de cancer du sein ou de l'estomac et les cystites chroniques ${ }^{5}$. En cas de doute sur le diagnostic, le recours à un examen immunohistochimique est indispensable. La positivité du marquage cellulaire aux anticorps anti-CK-7, CK-20, AE1/AE3, EMA et CD-138, associée à une négativité du marquage pour LCA, S100, HMB45, $\kappa, \lambda$ et CD79- $\alpha$, confirme le diagnostic de carcinome urothélial plasmocytoïde ${ }^{6-9}$.

Du fait de sa rareté, le traitement du carcinome urothélial plasmocytoïde est encore matière à débat. La maladie est généralement diagnostiquée à un stade avancé, comme l'indique le tableau 2. Pour traiter les formes d'emblée métastatiques, la plupart des auteurs proposent une chimiothérapie à base de cisplatine $\mathrm{e}^{7,10-13}$. Malgré une réponse initiale à cette chimiothérapie, les résultats restent insatisfaisants, puisque la médiane de survie globale est de 13,4 mois ${ }^{11}$. Pour les patients présentant une tumeur résécable non métastatique au moment du diagnostic, la cystectomie radicale, suivie d'une chimiothérapie adjuvante à base de cisplatine, semble le traitement le plus approprié.

Dans la série de Lopez-Beltran 7,7 patients traités par cystectomie seule sont décédés des suites de la maladie (dans un délai de 2 à 11 mois); 1 seul des 3 patients ayant suivi une chimiothérapie adjuvante après cystectomie est décédé de la maladie (au bout de 8 mois), alors que les 2 autres étaient en rémission (8 et 16 mois plus tard). Dans la série de Fritsche $^{10}$, le patient traité par cystectomie seule est décédé des suites de sa maladie après 14 mois. Deux patients de cette série ont reçu une chimiothérapie adjuvante : le premier vit toujours sans reprise de la maladie (à 16 mois), alors que l'autre est décédé (d'une embolie pulmonaire) après 29 mois. Enfin, Dayyani ${ }^{11}$ signale une médiane de survie globale de 45,8 mois chez 14 patients ayant reçu une chimiothérapie néo-adjuvante ou adjuvante à la cystectomie. Dans cette dernière série, les auteurs n'ont pas relevé de différence notable entre le délai de survie des patients ayant reçu une chimiothérapie néo-adjuvante et les patients traités initialement par cystectomie ${ }^{11}$. L'ensemble de ces résultats suggèrent un bénéfice potentiel de la chimiothérapie adjuvante pour les formes résécables non métastatiques. 
Dans notre série, le premier patient ne présentait pas de métastases ganglionnaires ou à distance, raison pour laquelle il n'a pas reçu de traitement adjuvant. Il est décédé un mois plus tard d'une embolie pulmonaire, probablement à la suite de la chirurgie pelvienne et carcinologique. Dans le deuxième cas, une chimiothérapie adjuvante a été entreprise en raison du caractère métastatique de la néoplasie. Le patient n'a reçu que deux cycles de chimiothérapie et est décédé deux mois plus tard. L'altération profonde de l'état général du patient est probablement la principale cause de décès.

\section{Conclusion}

Le carcinome urothélial plasmocytö̈de est une variante histologique rare du carcinome urothélial. La symptomatologie clinique n'est pas spécifique, et le diagnostic reste histologique. Le recours à l'étude immunohistochimique est indispensable pour le diagnostic. Celui-ci étant le plus souvent établi à un stade avancé, le traitement conseillé est la cystectomie, associée à une chimiothérapie adjuvante. Le pronostic à ce jour reste mauvais. Etant donné la rareté de ce type de tumeur, un consensus d'experts serait utile pour trouver la meilleure stratégie thérapeutique.

Competing interests: Dr. Benazzouz, Dr. Essatara, Dr. Elsayegh, Dr. Iken, Dr. Benslimane, Dr. Znati and Dr. Yassine Nouini all declare no competing financial or personal interests.

This paper has been peer-reviewed.

\section{Références}

1. Eble J, Sauter G, Epstein II, Sesterhenn IA, editors. Classification of tumours. Pathology and genetics of the tumours of the urinary system and male genital organs. Lyon: World Health Organization; 2004.

2. Sahin AA, Myhre M, Ro JY, et al. Plasmacytoid transitional cell carcinoma. Report of a case with initial presentation mimicking multiple myeloma. Acta Cytol 1991;35:277-80.

3. Keck B, Stoehr R, Wach $S$, et al. The plasmacytoid carcinoma of the bladder-rare variant of aggressive urothelial carcinoma. Int J Cancer 2011;129:346-54. http://dx.doi.org/10.1002/iic.25700

4. Billerey C, Sibony M. Tumeurs superficielles de vessie. Prog Urol 2001;11:826-37.
5. Rahman K, Menon S, Patil A, et al. A rare case of plasmacytoid urothelial carcinoma of bladder: Diagnostic dilemmas and clinical implications. Indian J Urol 2011;27:144-6. http://dx.doi.org/10.4103/09701591.78409

6. Ro JY, Shen SS, Lee HI, et al. Plasmacytoid transitional cell carcinoma of urinary bladder: A clinicopathologic study of 9 cases. Am J Surg Pathol 2008;32:752-7. http://dx.doi.org/10.1097/ PAS.0b013e318159af9e

7. Lopez-Beltran A, Requena MJ, Montironi R, et al. Plasmacytoid urothelial carcinoma of the bladder. Hum Pathol 2009;40:1023-8. http://dx.doi.org/10.1016/i.humpath.2009.01.001

8. Nigwekar P, Tamboli P, Amin MB, et al. Plasmacytoid urothelial carcinoma: Detailed analysis of morphology with clinicopathologic correlation in 17 cases. Am J Surg Pathol 2009;33:417-24.

9. Mai KT, Park PC, Yazdi HM, et al. Plasmacytoid urothelial carcinoma of the urinary bladder report of seven new cases. Eur Urol 2006;50:1111-4. http://dx.doi.org/10.1016/i.eururo.2005.12.047

10. Fritsche HM, Burger M, Denzinger $S$, et al. Plasmacytoid urothelial carcinoma of the bladder: Histological and clinical features of 5 cases. J Urol 2008;180:1923-7. http://dx.doi.org/10.1016/i.juro.2008.07.035

11. Dayyani F, Czerniak BA, Sircar K, et al. Plasmacytoid urothelial carcinoma, a chemosensitive cancer with poor prognosis, and peritoneal carcinomatosis. J Urol 2013;189:1656-61. http://dx.doi.org/10.1016/i. juro.2012.11.084

12. Kohno T, Kitamura M, Akai H, et al. Plasmacytoid urothelial carcinoma of the bladder. Int I Urol 2006; 13:485-6.

13. Raspollini MR, Sardi I, Giunti L, et al. Plasmacytoid urothelial carcinoma of the urinary bladder: Clinicopathologic, immunohistochemical, ultrastructural,and molecular analysis of a case series. Hum Pathol 201 1;42:1149-58. http://dx.doi.org/10.1016/i.humpath.2010.11.011

14. Zukerberg LR, Harris NL, Young RH. Carcinomas of the urinary bladder simulating malignant lymphoma. A report of five cases. Am J Surg Pathol 1991;15:569-76. http://dx.doi.org/10.1097/00000478199106000-00005

15. Gaafar A, Garmendia M, de Miguel E, et al. Plasmacytoid urothelial carcinoma of the urinary bladder. A study of 7 cases [in Spanish]. Actas Urol Esp 2008;32:806-10.

16. Mitsogiannis $I C$, loannou $M G$, Sinani $C D$, et al. Plasmacytoid transitional cell carcinoma of the urinary bladder. Urology 2005;66:194. http://dx.doi.org/10.1016/i.urology.2005.01.023

17. Aldousari S, Sircar K, Kassouf W. Plasmacytoid urothelial carcinoma of the bladder: A case report. Cases J 2009;2:6647. http://dx.doi.org/10.1186/1757-1626-2-6647

18. Sato K, Ueda Y, Kawamura K, et al. Plasmacytoid urothelial carcinoma of the urinary bladder: A case report and immunohistochemical study. Pathol Res Pract 2009;205:189-94. http://dx.doi.org/10.1016/i. prp.2008.09.004

19. Sakuma $T$, Furuta $M$, Mimura $A$, et al. Urine cytological findings of plasmacytoid urothelial carcinoma of urinary bladder: report of two cases. Cytopathology 2009;20:264-7. http://dx.doi.org/10.1111/ j.1365-2303.2009.00660.x

20. Shimada $K$, Nakamura $M$, Ishida $E$, et al. Urothelial carcinoma with plasmacytoid variants producing both human chorionic gonadotropin and carbohydrate antigen 19-9. Urology 2006;68:891, e897-810.

21. Soylu A, Aydin NE, Yilmaz U, et al. Urothelial carcinoma featuring lipid cell and plasmacytoid morphology with poor prognostic outcome. Urology 2005;65:797. http://dx.doi.org/10.1016/i.urology.2004.10.005

Correspondence: Dr. Mohamed Hicham Benazzouz, Service d'Urologie « A » Centre hospitalier universitaire Ibn Sina Rabat, Morocco ; benazzouzhicham@gmail.com 